\title{
Sound2Picture: Developing compositional pedagogies from the sound designer's world
}

\section{Jonathan Savage (BA hons, PGCE, PhD)}

Email: j.savage@mmu.ac.uk

JONATHAN SAVAGE is a Senior Lecturer in Music Education at the Institute of Education, Manchester Metropolitan University. Until 2001 he was Head of Music at Debenham High School, an 11-16 comprehensive school in rural Suffolk. His main research interests lie in the field of developing innovative uses of new technologies within the music curriculum, particularly in promoting new approaches to composition. He is co-author of the Sound2Picture resource that illustrate how sound design can be applied within the music curriculum. 


\begin{abstract}
Sound design is one example of a current artistic practice dominated by the computer and other digital technologies. Recent research evidence from ImpaCT2 has noted that it is a worthy goal to integrate new technologies with subject learning (DfES 2002, p.3). Sound design offers an exploration of exciting new notions of artistic practice that integrate rich mixes of subject learning with new technologies. It was hypothesised that this could help develop music education towards a holistic model of artistic practice mediated through the effective use of new technology.

Sound2Picture was the latest stage in a series of research and development projects being conducted at Manchester Metropolitan University (MMU). It documented the work of one sound designer who demonstrated a host of new skills and imaginative practices with hardware and software. The article shares aspects of this work through case study and applies the ideas therein to the work of music educators.
\end{abstract}




\section{Introduction}

Sound2Picture was the latest stage in a series of research and development projects being conducted at Manchester Metropolitan University (MMU). These began in 2002 with a grant from Palatine and an investigation into the songwriting practices of popular music composers (Savage 2003). During this period MMU researchers met up with a team from the Virtual Learning Environments Foundation who were collaborating with Yamaha Kemble on a project entitled Found Sound. This resulted in Yamaha Kemble's production of a CDROM of resources that is still freely available and available direct from Yamaha Kemble $^{1}$.

Through the research dimension of this songwriting project, collaboration between MMU and Alchemy Audio Lab was established, in particular with the lead sound designer Andrew Diey. The Sound2Picture CDROM was the first product of this collaboration (www.sound2picture.net).

Alongside the production of this CDROM of teaching resources, MMU researchers conducted a parallel piece of research into the sound design process. This research is described in the following article. But before this dimension of the project is introduced, an introduction to sound design for those unacquainted with the term is well overdue.

\section{What is Sound Design?}

Sound designers guide the sound of a motion picture from beginning to end, interpreting the director's expressions, "hearing" the script and storyboards, coordinating with the composer and sound editor, contributing to the mixing process, even ensuring that what is heard in the theatre is of optimum quality. (Mancini in Weis \& Belton 1985, p.361)

Mancini's description of the sound designer's role is a good starting definition. As the amount of visual information contained with movies has increased so audiences must have correspondingly detailed and heightened sound effects in order to perceive these images and sense them as realistic. The soundtrack of a movie (containing the musical score and sound effects) is therefore every bit as important as the visual element. As Thom points out:

You may assume that sound design is about fabricating neat sound effects. But that doesn't describe very accurately what Ben Burtt and Walter Murch, who invented the term, did on Star Wars and Apocalypse Now respectively. On those films they found themselves working with Directors who were not just looking for powerful sound effects to attach to a structure that was already in place. By experimenting with sound, playing with sound (and not just sound effects, but music and dialog as well) what they found is that sound began to shape the picture sometimes as much as the picture shaped the sound. The result was very

${ }^{1}$ From Karen Watts on 01908369253 
different from anything we had heard before. The films are legends, and their soundtracks changed forever the way we think about film sound. [my italics]

(Thom 1999. p.1)

\section{The Practice of Sound Design}

Sound design is attracting more attention now than it ever has done, primarily due to great sound design in movies, computer games and on our television screens. But the increasing availability of computer hardware and software has meant that what was previously the domain and practice of the professional studio composer can now be done by anyone possessing a modestly equipped personal computer and access to the Internet.

The aim of the Sound2Picture research was to illustrate how the processes and practices of sound design, i.e. creating, classifying, editing and mixing sounds to picture, can extend existing school-based compositional pedagogies.

\section{The Educational Potential of Sound Design}

But why consider the work of sound designers in order to inform the teaching of composition in high schools? What was the hypothesised potential of sound design for music education? There were two main reasons.

Firstly, that the relationship between music and the visual image are long established in artistic practice but, by comparison, has often been neglected in the recent history of music education. Secondly, the role of digital technologies within schools is becoming increasingly pervasive and that these technologies demand and facilitate new ways of working within and across the arts. The practice of sound design contains some wonderful applications for the uses of these new technologies.

\section{The Painting of Sound}

Composers and painters alike have frequently gleaned new ideas from an approximation to, or borrowings from, procedures used in the sibling art. This reciprocal relationship runs like a continuous thread through the entire [twentieth] century. (Maur 1999, p.8)

This quotation illustrates the close relationships between artists and composers in recent Western history. Perhaps one of the most famous and obvious examples of a painter considering musical themes is Paul Klee, for whom music 'was the one discipline of art above all others that inspired profound insight' and showed him 'the innermost essence of nature, not a reproduction of it' (Düchting 2002, p.88). But throughout the entire twentieth century the symbiotic artistic processes and conversations between the visual artist and composer are apparent for all to see (and hear). So it is no surprise to find 
philosophical and religious beliefs about the interdependence of sound and colour through the compositions and writings of many, including Ciurlionis and Kandinsky:

There are no dividing walls between the arts. Music combines within itself poetry and painting and has its own architecture. (Ciurlionis 1998, p.53)

Colour is the key, the eye is the hammer, the soul is the piano with its many strings. (Kandinsky 1947, p.64)

As Maur has outlined his argument for 'the sound of painting', within Sound2Picture sound design can be conceived as 'the painting of sound'. It was not surprising that sound designers talked about their work with sounds by way of visual metaphors. For example, Diey talks about the brush strokes of his compositional style:

I feel that sound design is an area in which you can either paint with very large strokes or very fine strokes. You can go as deep as you like and put as much detail in as required. Or you can just paint with broad strokes. (Andrew Diey in interview, 2004)

or the relationship between the choice of sounds and particular colours:

The overall feel and colour of the movie is washed out colours, lots of blues and dark sort of deep browns and things like that. So it automatically suggests a sort of cold feel to the actual sound itself. There's not much warmth in the colour so I have to reflect that in my choice of sound. (Andrew Diey in interview, 2004)

\section{The Artistic Potential of New Technologies}

The second reason that sound design was chosen as a focus for this research was that recent research has shown the interdependence of artistic work when new technologies are incorporated within the classroom environment (Savage \& Challis 2001, 2002). One of the main themes within the research was the interplay between aural and visual work, in particular that each associated 'discipline' can be used to reinforce the thinking, creative ideas, potential and understanding of the other and beyond to other parts of the curriculum.

Sound design offers the potential to facilitate more of this cross-disciplinary interchange within the 'seductive meeting point' of new technologies (Waters 1994, p.28).

Researchers perceived that it might help bridge the gap between what can be disparate artistic practices within schools. Conceptually, this requires a redefinition of a subject's culture and working practices, as well as a consideration of how these may relate to other parts of the curriculum. Francis Dhomont, the classical French electroacoustic music composer, summed it up like this: 
We have more in common with the filmmaker or the sculptor, the painter, with the plastic artist, than with the traditional musician. I really have that feeling, even though my origins are in traditional music. (Dhomont 2002)

New technologies radically transform 'the arts' in ways that are slowly beginning to be understood. Recent research evidence from ImpaCT2 has noted that it is a worthy goal to integrate new technologies with subject learning (DfES 2002, p.3). Sound design offers an exploration of exciting new notions of artistic practice that integrate rich mixes of subject learning with new technologies. It was hypothesised that this could help develop music education towards a holistic model of artistic practice mediated through the effective use of new technology.

\section{The Research Methodology}

Sound2Picture adopted a research method that was qualitative in design. It focussed on the work of one particular sound designer, Andrew Diey, identified during the previous Found Sound project, whose approach to sound design seemed particularly interesting. He was also selected because of his ability to talk coherently about his musical products and describe, often in intimate detail, the working process that he was adopting.

Case study of this type has a long tradition within the United Kingdom's educational system including Treacher (1989 \& 1992) who used action research and case study to produce curriculum specifications and materials. A more recent adaptation of this approach has been used by Sefton-Green in his survey of the digital arts (Sefton-Green 1999).

Robert Stake and Lawrence Stenhouse define case study as:

The collection and recording of data about a case or cases, and the preparation of a report or a presentation of the case. (Stenhouse 1988, p.49)

The study of the particularity and complexity of a single case, coming to understand its activity within important circumstances. (Stake 1995, p.xi)

Stake emphasises the importance of studying in detail the particularity and complexity of a single case. This seminal text on the art of case study research is constructed around an actual case study that he conducted at Chicago's Frances Harper Elementary School. But Stenhouse is quick to point out that case study can also be generalised and comparative in its design:

Many researchers seek theories that will penetrate the varying conditions of action, or applications founded on the comparison of case with case. (Stenhouse 1988, p.49) 
The exact balance between studying individual cases or generalisations across cases are matters for the case study researcher's judgement. But either way, case study should produce ordered reports of experience that 'invite judgement and offer evidence to which judgement can appeal' (Stenhouse 1988, p.49). In this sense, Stenhouse suggests, case study is like history in that its appeal to judgement rests on the representation of events and persons within these events. The process by which one seeks to represent people and events within one's case studies is therefore highly significant.

Within the practice of case study there are several main styles. Stenhouse (1988, p.49) identifies four principle types of which this research can best be categorised as ethnographic case study. This is a single in-depth case investigated primarily by participant observation supported by interview.

\section{The Research Method}

The main research methods used throughout this research were participant observation and interview. Additionally, a range of other documentary materials was carefully analysed including multimedia materials and a selected bibliography of other sound designer's work.

\section{Participant observation and interviewing}

Close and thoughtful observation of others is always an important and intensive feature of participant observation, and this includes, as an important element, observation of speech in natural settings. (Stenhouse 1988, p.51)

During the research a number of formal observations of Andrew's work were made whilst he composed sound designs for six short movies. For a brief description of each movie and a collection of screenshots please see Appendix A. These observations were videotaped and, on occasions, involved Andrew giving a 'commentary' of his working

process. In addition, Andrew kept detailed notes of his working process and a selection of screenshots from his computer to illustrate the various stages of each composition's development. At the conclusion of the project Andrew was interviewed about his work. The transcript of this interview contained much valuable information and can be read in full at the project website (www.sound2picture.net). The data drawn from this interview and observations were collated and analysed using basic principles drawn from grounded theory (Glaser 1999). 
2. The collection and analysis of other documentary materials, including photographs, video and music

The multimedia nature of the case study meant that many other materials were developed throughout the project. Musical outcomes were particularly significant as were photographs and video of Andrew's work. Many of these materials found their way into the final CDROM of teaching materials but also proved very interesting from the research perspective.

Finally, the organisation of all the materials generated by a case study is a crucial part of the process of analysis and needs to be managed effectively. The case record generated by the Sound2Picture research was large and needed to be handled in such a way that key concepts and ideas were not lost. Given the multimedia dimension of the case study, Walker's notion of representation and presentation was particularly significant (Walker 2002 , p.117). Drawing on the work of Stenhouse (1978, p.37) Walker differentiates the process of representation, by which he means the way in which data is represented for use by researchers, to presentation, which is the 'data organised in order to present to a reader an interpretation of its significance in relation to some topic, issue or problem' (Walker 2002, p.117).

It is under this notion of presentation that this article will present selected findings from the represented data that constituted the Sound2Picture case study. These findings will be considered within a description of the working process that Diey followed throughout the Sound2Picture research.

\section{A Sound Design Process}

Diey provided detailed notes describing how he approached each of the six movies within the Sound2Picture project. These notes provided a fascinating insight into how he worked as a sound designer. The process of work in each movie was slightly different but common themes emerged. This following table outlines the main steps as Diey identified them:

\begin{tabular}{|l|l|}
\hline Step 1 & Thinking in Pictures \\
\hline Step 2 & Choosing your colour palette \\
\hline Step 3 & The Source: Choosing your overall sounds \\
\hline Step 4 & The Visual Cue: Choosing specific sounds \\
\hline Step 5 & Additional Elements \\
\hline Step 6 & The Final Mix \\
\hline
\end{tabular}


Other recent research has identified a clear process of composition drawn from observations of the work of postgraduate composers at the University of East Anglia (Savage \& Challis $2001 \& 2002$ ). This compositional process was used to underpin the work in two major case studies of pupils' work with digital technologies and can be illustrated as:

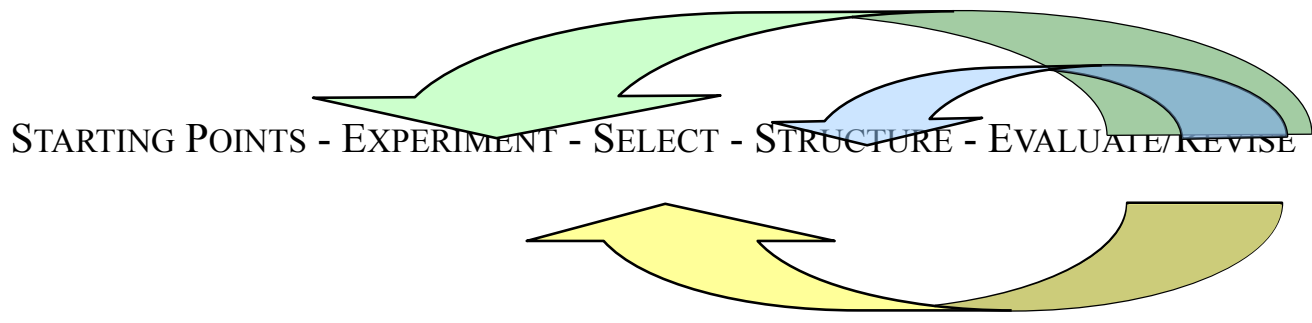

A combination of these two models gives the following process:

\begin{tabular}{|c|c|c|}
\hline Step 1 & Thinking in Pictures & \multirow{2}{*}{ StARTING POINTS - } \\
\hline Step 2 & Choosing Your Colour Palette & \\
\hline Step 3 & The Source: Choosing your overall sounds & \multirow{3}{*}{$\begin{array}{l}\text { EXPERIMENT - } \\
\text { SELECT }\end{array}$} \\
\hline Step 4 & The Visual Cue: Choosing specific sounds & \\
\hline Step 5 & Additional Elements & \\
\hline Step 6 & The Final Mix & STRUCTURE \\
\hline
\end{tabular}

\section{Step 1: STARTING POINT 'Thinking in Pictures'}

A key feature of Diey's work was his ability to 'think in pictures'. During interview he explored the potential of the moving image as a source for inspiration in his sound design. What did 'thinking in pictures' mean? For Diey, it came down to two main things.

\section{a. Environmental Issues}

Firstly, Diey asked key questions about the environmental perspectives that are played out within the movies. He considered how these perspectives were defined and what they might mean. Sometimes the environmental perspective was clearly defined as in the Citydrive movie: 
So within the actual perspective if you look at the physical location of the sounds that are required you're sitting in a traveling automobile so you are already defined within the borders of your acoustic environment. (Andrew Diey in interview, 2004)

In this example the physical environment was fairly fixed and this led to an unproblematic assumption as to which elements of the acoustic environment would need to be considered within the sound design:

And so straightaway you can start to break down the elements of what you will immediately hear ... (Andrew Diey in interview, 2004)

But what about when things were not quite so obvious from a physical environmental perspective? The Graphix movie was very different from the other movies in this respect. It contained a series of very colourful but mostly abstract pictures and images. This presented a different dilemma:

It's kind of hard to nail down exactly what the pictures will sort of say from a sound perspective because there is no real visual hook other than the key elements that are on these, such as motion, colour and movement. (Andrew Diey in interview, 2004)

In the absence of a direct physical environment other elements came into play. These were not always related to the visual image but included musical forms and devices, a point discussed further below.

The consideration of a movie's environmental perspective led Diey to make a series of choices that affected all the subsequent steps of the sound design process. In looking for inspiration from the environment presented in a sequence of film, he had to consider certain fundamental questions such as:

- How had the physical environment been defined?

- Were there aspects of the suggested physical environment that gave clues as to the possible accompanying acoustic environment that he was going to create?

- Should the sound design embrace or ignore, restrict or extend the possible acoustic environments suggested by the pictures?

- Could the visual environment be transformed through a new approach to the acoustic environment that would be created?

As with each step of the sound design process, Diey made creative choices here. These choices had important consequences for the final shape of the sound design. But it is important to emphasise that he did not see these as right or wrong responses. There were multiple interpretations of the environmental features of any given movie and, as the two versions of the sound designs for the Butterfly movie demonstrated beautifully, the end results were very different. 


\section{b. Interrogating and Exploring the Visual Image}

The second main feature of 'thinking in pictures' was the direct and detailed interrogation and exploration of the visual image. There were numerous examples of this within each of the six movies. The critical point is that key visual elements or cues inspired a response from the sound designer:

The Nature Technology movie itself is really quite stark. The feel of the sounds within the piece are two fold. You have the small, discrete sounds of nature and then you have the big bold sounds of the radio telescope that have an almost cold war feel about them. The way that the movie is shot is eerie and almost with a sense of suspense to it so the sounds that I chose initially were big, underlying, slow moving, discordant chords which then evolve into another discordant chord. (Andrew Diey in interview, 2004)

At a certain level there was a basic following of the visual events of the movie (drawn from this interpretation of the visual image). But at a deeper level Diey considered the elemental features of images as a source for inspiration. This became particularly important when the images on screen were more abstract. Once again, the Graphix movie gave the best demonstration of this:

The graphics themselves are full of colour, motion and depth. There's not really much to be said about the meaning behind the visuals. So when you get a piece of abstract visuals, it's often best to rely on musical form to carry the mood forward. So the choices that I made for this piece of graphics was to put a music backing track and try to latch on some of the motion that is actually in the movie itself, whether it's a piece of graphics that is actually shooting past the screen, whether it's a slow moving piece of graphics. (Andrew Diey in interview, 2004)

Diey linked the abstract nature of the visuals with the familiarity of musical form in order to carry the mood of the movie forward. What could be described as Diey's compensatory approach to the movie allowed him to imbue abstract visuals with a musical sense of motion and progression that carried them to a point beyond that which they could have reached independently of the sound design. It was a good example of abstract visual elements affecting music choices that did, in turn, lead to the embracing of musical opportunities.

\section{Step 2: STARTING POINT 'Choosing Your Colour Palette'}

One of the most important assets of a sound designer is their sample library:

Categorisation of sounds is primarily a personal and subjective way of collecting and organising sounds. One of the most important parts of being a working sound designer on a day-to-day basis is the ability to know your sound library. (Andrew Diey in interview, 2004) 
Diey's sound library is a personal collection of over $1 \frac{1}{2}$ terabytes of original sounds that have been recorded and collected over many years. Such a massive amount material requires careful classification and archiving. Diey could save valuable time by being able to place his hands (and ears) on a particular sound file quickly and easily.

Diey's first skill was being able to classify sounds into various colour palettes that might relate to his particular thoughts about the visual images contained within a movie.

The order in which my sounds are categorised are often into types of sound whether they're nature, machines, abstract, electronic, synthetic, musically applied sound effects or acoustically recorded sound effects. (Andrew Diey in interview, 2004)

But things were slightly more complicated than just choosing one category for a specific sound:

If I take, for example, the sound of a gong, a huge 20" gong, it can be recorded in many different ways such as contact recording or air-born recording. The usage of that gong is quite diverse. You can take the sound of the gong and put it into a musical frame. But you can use it such as the Nature Technology movie at the end, where I EQ-ed filtered sounds to give the sound of punctuation. (Andrew Diey in interview, 2004)

So Diey's second skill in choosing a colour palette was to realise the diverse potential of sounds, even those that may not be the initial and most obvious choice. Sound designers have an acute sense of their sound environment and the potential of sounds that many of us might consider insignificant as musical material. Developing this interest in sound was a vital skill:

Sound design is very much a case of being aware of what is around you in your environment. Any sound designer who works professionally will tell you that the most important tool for a sound designer is their ears. Secondly you have your auxiliary ears known as your microphones. And thirdly you have the recording device which is the DAT machine, tape machine or Minidisc. You must learn to pay attention to what sounds are around you. (Andrew Diey in interview, 2004)

\section{Step 3: EXPERIMENT - SELECT 'The Source: Choosing your overall sounds'}

For Diey, as with any composer, the whole composition process is about exploratory play. Our observation of Diey's work suggested that within this approach there were various discrete steps or stages of work. But it is important to remember that every step was characterised by making choices and that these choices had consequences.

When asking Diey about the particular sounds that he had chosen for each movie it soon became apparent that he thought about the layers of sound within each sound design. 
From this we decided to conceptualise his choices against the categories of 'overall sounds' and 'specific sounds'. The concepts are self-explanatory but they facilitated a detailed consideration of how and why particular sounds were chosen for each composition.

As an example, Diey's comments on the Butterfly movie were particularly informative:

If we look at the sounds as two or three layers you have the foreground which is the sound of the butterfly itself as it moves around the screen. And then you have the background sounds of the daytime and what that might mean in this garden region. The background sounds that I chose to put in were a couple of layers including the sounds of wind, traffic passing and, most importantly, the sounds of children playing in the background. (Andrew Diey in interview, 2004)

For Diey, one particular background sound had particular significance - the sound of children playing with butterflies. This took him right back to his childhood and his frequent visits to butterfly farms in Edinburgh.

When I was younger I visited a butterfly farm just on the outskirts of Edinburgh. It was in this enormous heated cocoon and there were hundreds of butterflies from exotic ones to the more common Red Admiral and things like that. I always liked it because of the colour and the way that these creatures moved. . (Andrew Diey in interview, 2004)

However, its inclusion developed an important point for any sound designer - the preeminence of one's own personal life experience as a key factor in the composition process. To demonstrate this point, Diey completed two distinct sound designs for the Butterfly movie. One is a reflection on his childhood and the other reflects on his wider work in music over the past fifteen years.

My experiences have been very much involved in dance music, electronic music, having record shops, being on radio shows and working on a daily basis as a professional composer. I decided to put lots of energy and musical energy within the actual butterfly movement themselves and the choices that I made were to put beats underneath and carry the movement forward. (Andrew Diey in interview, 2004)

Overall sounds, whether they are the sound of children playing or contemporary beats, have a vital role to play in the sound design. They act as a 'sound bed' against which the choices of specific sounds can be contrasted.

\section{Step 4: EXPERIMENT - SELECT 'The Visual Cue: Choosing your specific sounds'}

In Butterfly movie take one there are about seven layers of sounds. There is the protagonist which is the butterfly himself, and he is the main focus. So I followed the sound around the screen. The way that I created the sound of the wings flapping was quite a synthetic way. I took a very quick repeated sound to mimic 
the movement of the wings as the butterfly carries itself around the actual frame. And then I added movement to the actual sound. This is known as modulation and within music it is a very common type of effect to give a sense of movement and dynamic to an actual sound. Within this I decided to follow the movement of the wings by choosing volume curves and panning effects. So I decided that because the butterfly was so beautiful I had to follow it as the camera had followed it around the screen. (Andrew Diey in interview, 2004)

This part of the interview showed how much detail Diey applied to one particularly significant sound in the Butterfly movie. The careful treating of sounds in this way is an important next step in the sound design process.

In a similar way, the Futurescape movie gave Diey the opportunity to tightly knit together particular events on the screen with specific sounds:

It is very easy to see why I chose certain sounds for some of the graphics on the screen. Because there are graphics that come towards you and have a certain sense of motion and the camera quickly turns the corners. These types of visual cues are just delightful to work for from a sound and music point of view it gives you a point from where to turn the music or where to add the sounds or how quickly to put the sounds in or make them fade out. (Andrew Diey in interview, 2004)

But how did he know whether or not two sounds would work well together?

A lot of that is down to experience really. But if you are starting out the important thing is to experiment and see what happens when you put two sounds together. (Andrew Diey in interview, 2004)

The importance of experimentation prior to selection is one of the resounding themes of the interview with Diey and the observations of his work. It is through this process of experimentation that he was able to achieve 'happy accidents:

It is often the case that you'll get happy accidents. The way to get happy accidents is to throw things at each other and see if they work. And once you see that certain things do work you'll know that in the future when you do come to do another set of visuals you can have these two elements working together. Experiment, experiment, experiment and learn your craft. That's what it's all about. (Andrew Diey in interview, 2004)

But what did he do when two sounds did not work together?

There are a couple of cases where a sound just won't work. Either the sound is completely wrong or the sound needs a little bit of work done to it. The vast array of sound treatments that you can work with sounds vary from equalisation, filtering, pitch-shifting, etc. Within these basic processing types you have a million different options. If the sound doesn't work and you keep trying to make it work then just get rid of it. Use another sound. It's very simple just dump the sound. If something doesn't work after you've tried several times then move onto 
the next piece. It's very simple. Not all sounds do work together. Not all sounds are meant to work together. Experiment. [my italics] (Andrew Diey in interview, 2004)

\section{Step 5: EXPERIMENT - SELECT 'Additional Elements'}

The penultimate stage in the sound design process was the inclusion of additional elements into a particular sound design. In Diey's work these elements took a number of forms including the addition of:

- Specific musical events or elements within the general soundscape of the sound design;

- Particularly important sound events that could potentially change the entire dynamics of the sound design;

- Discrete but powerful ranges of digital effects to all or part of the sound design.

\section{a. The inclusion of specific musical events or elements}

The second Butterfly sound design is perhaps the best illustration of the inclusion of additional elements. Within this sound design Diey chose to add a powerful rhythm section and drone to the sounds of the butterfly movements. This completely changed the dynamic of the sound design.

So you still have the movement of the wings laced on top with the sounds of a very, I wouldn't say aggressive, but more powerful sounding chords underneath that. There is a certain sense of dynamic and movement and structure and the sounds were just sort of carrying the butterfly along, giving the butterfly a sense of urgency without compromising what his daily duties were. (Andrew Diey in interview, 2004)

This incorporation of musical elements allowed Diey to interpret the same movie in a very different way from the naturalistic portrayal discussed above. He conceived this version as representing the adult perspective of a professional sound designer as opposed to the initial version from a child's viewpoint:

The Butterfly Take 2 movie is very much a contemporary, up-to-date version of how I feel about music and sound. The perspective that I have nowadays compared to when I was a child as in the first movie you could say is twenty years on with a whole plethora of twenty years experience of being in a very enriched music world. (Andrew Diey in interview, 2004)

b. The inclusion of particularly powerful sound events that have the potential to change the entire dynamic of the sound design

Diey's interpretation of the Citydrive movie was from the first person perspective of travelling through a changing cityscape in a moving automobile. This perspective made 
obvious demands on him as a sound designer, not least the inclusion of sounds from the automobile:

So my choices for the immediate acoustic environment were to look at the car and the sounds the car will make. So I chose a varied selection of car engines to give the emphasis on what type of car we are in. (Andrew Diey in interview, 2004)

The choice of car sound and the way in which it was mixed within the sound design had important consequences. Diey's definition of his environmental perspective allowed him to make clear choices about other sound events that can be included or precluded from his sound design:

Because we are within a car there is a perfect environment in which to ignore the sounds outside or to embrace the sounds outside. You could imagine listening to music or to having the windows open and enjoying the sounds that are coming from the exterior. The choices I made were to try and bridge the gap between being isolated within a car and including yourself within the actual city environment. (Andrew Diey in interview, 2004)

The imaginative extension of this first person perspective was a key component of a creative approach to working with sound and picture. The ability to imbue certain sound events with significance beyond the obviously semantic was a true characteristic of 'painting in sound'.

\section{c. The inclusion of discrete but powerful ranges of digital effects to all or part of the sound design}

There are huge and powerful ranges of digital effects that can be added to individual sounds (akin to small brush strokes) or applied to the whole composition (in broad brush strokes) as part of the final mix. The use of such digital effects is, in itself, an important part of the compositional or artistic process. Diey discussed the use of such effects within particular sound designs, e.g. the huge metallic structure of the radio telescope in Nature Technology ...

the way that it moves and the way that when it does move it moves with slow and bulky sounds. So a lot of the sounds in there are pitched down with reverb to give the impression of very slow moving metallic structures. (Andrew Diey in interview, 2004)

As with the butterfly's wing and the modulation effect, volume curves and panning effects that were applied for maximum effect it became apparent that Diey applied digital effects for particular artistic reasons. Whilst an experimental approach to processing sounds quickly produced the most amazing range of sound effects, he was able to make artistic choices and provide good explanations as to what he was attempting to do and why he chose particular courses of action to achieve this. 


\section{Step 6: STRUCTURE - EVAlUate/REVISE 'The Final Mix'}

The final section of this analysis of Diey's compositional process is the structuring together of ideas into a final, linear sound design that then needs to be reduced from its composite tracks into a final stereo mix:

The mixing down process in sound design is very similar to mixing music or very similar to DJing. You have one record here, you have another record here and you mix the two sounds together. Sometimes the beats match and sometimes they're out of synchronisation and you need to match them up. Mixing down is very much an art form that people have perfected over the years and it only comes with experience and playing around with how the sound levels work together. What I would suggest to continually try to experiment with different types of mixes on your overall piece of audio. (Andrew Diey in interview, 2004)

His final point is perhaps the most important to consider. The concept of experimentation applies here just as it has done throughout the whole compositional process.

\section{Conclusion}

The development of computer technology has suggested to many that the computer can become a technology of unparalleled importance in the arts. What does such a vision imply for the creation of school programs in the arts? What does a computer allow students to do with images that other technologies don't, and what might such a resource mean for the development of cognitive skills?

(Eisner 2002, p.41)

Eisner's challenge to art educators faces those within the music education community with equal relevance and force. Important decisions will need to be made about the future purpose and practice of music education in light of this technological revolution. But for many the focus has been elsewhere:

For many schools the main focus of activity following installation of networked ICT infrastructure was on teaching ICT skills. Cross-curricular use of ICT is difficult for secondary schools to achieve because ICT has traditionally been a specialist subject for GCSE. A major shift in culture and established practice is involved in the introduction of ICT within subject teaching. [my italics] (DfES 2002, p.19)

Sound design is one example of a current artistic practice dominated by the computer and other digital technologies. Within the work of a sound designer are a host of new skills and imaginative practices with hardware and software that have tremendous potential for those educators seeking that 'major shift in culture and established practice'. 
Eisner's comments about the potential and challenge of the computer come in a chapter entitled 'Visions and Versions of Arts Education' which seeks to describe some of the common aims and content of arts education programs common in schools today. He states clearly that there is no 'sacrosanct vision of the aims of arts education' and that 'examples of diversity abound' (Eisner 2002, p.25). However, Eisner's summary of the five principles of arts education helps to explain the application of sound design to the educational practice

\section{Art education should give pride of place to what is distinctive about the arts}

Sound design is a distinctive and contemporary artistic practice that has recently become dependent on new technologies. It contains a range of artistic skills, often specific to the uses of new technologies that widen the opportunity for pupils to engage in musical composition. For these reasons, if nothing else, sound design is worthy of inclusion in the music curriculum.

\section{Art education programs should try to foster the growth of artistic intelligence}

Ability in art is assigned to talent, ability in 'intellectual subjects' is assigned to intelligence. (Eisner 2002, p.43)

Intelligence takes many forms. Sound design exhibits peculiar forms of artistic and musical intelligence within the cultural context of an enriched technological environment. Intelligent evaluation and reflection on the process of creation is a common theme in Diey's work. At times it is reflection on the visual image, its place in the movie and its relationship to other visual elements; on other occasions it is reflection on the purpose, function and expressive affect of sonic events. Either way, intelligence belongs to the arts just as much as to any other part of the curriculum.

\section{Art education programs should teach how to create and experience the aesthetic}

Eisner's suggestion is that within curriculum development the arts 'can, and probably in most situations will, be addressed in an integrated fashion' (Eisner 2002, pp.43-44). Sound2Picture has shown that sound design allows pupils to jointly consider aspects of visual and musical significance and investigate their relationships within the digital medium. Pupils have now got another opportunity to create and experience the aesthetic through an integrated model of arts education. 
4. Art education should help pupils recognise what is personal, distinctive and unique about themselves

Personal responses are key to all arts education. Diey has provided examples of this through Sound2Picture, especially with his work on the Butterfly movie. A pupil's personal response to a movie is the key to his or her own creative work in this medium. Starting points are vital in achieving depth and quality in this kind of work. Creating educational situations in which pupils can imbue their personality, character and creative spirit is the key for teachers. Sound2Picture is just a starting point for this work.

5. Art education programs should make special efforts to enable pupils to secure aesthetic forms of experience in everyday life

Each subject studied in schools affords the student a distinctive window or frame through which the world can be viewed. (Eisner 2002, p.45)

Finally, Eisner draws attention to the larger issues that arts educators hopes to address, i.e. facilitating in pupils an appreciation of the wider world as a rich source of meaning when viewed within an aesthetic frame of reference. This is a lofty ideal to which no single curriculum resource or piece of research can claim exclusive rights.

But in an alternative sense, the work done by Diey in the Sound2Picture project is materially linked to the every day. The raw materials of his craft are the found sounds that have been recorded, manipulated and mixed together in the digital environment. The treatment of everyday materials as potential artistic objects has long been recognised as an artistic opportunity by some:

I believe that the use of noise to make music will continue and increase until we reach a music produced through the aid of electrical instruments which will make available any and all sounds that can be heard. ... The present methods of writing music will be inadequate for the composer who will be faced with the entire field of sound. (Cage 1968, p.4)

In typically provocative style, John Cage neatly summarised what he saw as the inadequacy of traditional methods of music making in light of the changing conceptual basis on which musical materials are defined.

There is an increasing need for music teachers to make links across the curriculum, in particular with the other performing or visual arts. Sound design contains a rich history of collaboration between the various members of a film studio. But there is a much longer history of collaboration between the visual artists and composers that clearly shows the interdependence of both art forms in terms of final products and, more importantly, the artistic process 
Finally, there is a strong argument for teachers to respond to the natural pull of digital technologies towards the digital arts (Sefton-Green 1999). The creative use of new technologies can resituate musical practices within the world of the digital arts. Sound2Picture has highlighted that an integrated arts or multimedia approach to sound design and composition may well engage and motivate pupils more successfully, facilitate the development of their creative skills and bring about the personal aesthetic awareness that we all seek. 


\section{References}

Cage, J. (1968) Silence Cambridge, Massachusetts, M.I.T.

Ciurlionis, M. K. (1998) M. K. Ciurlionis (exhibition catalogue) Cologne, WallrafRichartz-Museum.

Department for Education and Skills (DFES) (2002) Pupils' and Teachers' Perceptions of ICT in the Home, School and Community London, DfES.

Dhomont, F. (2002) My Cinema for the Ears (DVD) Bridge Records Inc.

Düchting, H. (2002) Paul Klee: Painting music London, Munich \& New York, Prestel.

Eisner, E. (2002) The Arts and the Creation of Mind New Haven \& London, Yale University Press.

Glaser, B. G. (1999) The Discovery of Grounded Theory: Strategies for qualitative research New York, Aldine de Gruyter.

Kandinsky, W. (1947) Über das Geistige in der Kunst New York, Wittenborn, 1947.

LoBrutto, V. (1994) Sound-On-Film: Interviews with creators of film sound London, Praeger.

Maur, K. (1999) The Sound of Painting: Music in modern art London \& New York, Prestel.

Savage, J. (2002) 'New Models for Creative Practice for Music Technologies' in NAME How Are You Doing? Learning and assessment in music Matlock, National Association of Music Educators (NAME).

Savage, J. (2003) Informal Approaches to the Development of Young People's Composition Skills, Music Education Research 5 (1), pp. 81-85.

Savage, J. \& Challis, M. (2001) Dunwich Revisited: Collaborative composition and performance with new technologies, British Journal of Music Education 18 (2), pp. 139-149.

Savage, J. \& Challis, M. (2002) A Digital Arts Curriculum? Practical ways forward, Music Education Research 4 (1), pp. 7-21. 
Sefton-Green, J. (1999) (ed.). Young People, Creativity and New Technologies: The challenge of the digital arts London, Routledge.

Stake, R. (1995) The Art of Case Study Research London, Sage Publications.

Stenhouse, L. (1978) Case study and Case Records: Towards a contemporary history of education, British Educational Research Journal 4 (2), pp.21-39.

Stenhouse, L. (1988) 'Case Study Methods' in Keeves, J. P. (ed.) Educational Research, Methodology and Measurement: An international handbook Oxford \& New York, Pergammon Press, pp.49-53.

Thom, R. (1999) Designing A Movie For Sound (http://www.filmsound.org/articles/ designing_for_sound.htm) [Accessed on 5/10/04]

Treacher, V. (ed.) (1989) Classroom Issues in Assessment and Evaluation in the Arts: The results of a collaborative action research project 1986-1988 Reading, Berkshire LEA.

Treacher, V. (ed.) (1992) Behind the Debate: The impact of the National Curriculum in the Arts Reading, Berkshire LEA.

Varese, E. (1967) 'The Liberation of Sound' in Schwartz, E. \& Childs, B. (eds.)

Contemporary Composers on Contemporary Music New York, Holt, Rinehart \& Winston.

Walker, R. (2002) Case Study, Case Records and Multimedia, Cambridge Journal of Education 32 (1), pp.109-127.

Waters, S. (1994) Living Without Boundaries Bath, Bath College of Higher Education Press.

Weis, E. \& Belton, J. (eds.) (1985) Film Sound: Theory \& Practice New York, Columbia University Press.

\section{Websites}

Alchemy Audio Lab: www.alchemyaudiolab.com

Sound2Picture: $\underline{\text { www.sound2picture.net }}$ 



\section{Appendix A: Movie Descriptions and Images}

\section{Movie 01: Citydrive}

Citydrive is a first person perspective from the interior of a moving car. The themes are cities, people and motion.

Insert:

movie01a.jpg

movie01b.jpg

\section{Movie 02: Nature Technology}

Nature Technology takes two opposing entities and puts them side-by-side in film. On one side there is nature (plants, animals, weather) and on then other technology (communications, structures, cameras).

Insert

movie02a.jpg

movie02d.jpg

\section{Movie 03: Butterfly}

Butterfly follows a butterfly on its daily duty of pollinating flowers and hovering gracefully in the process. It is also entirely shot on the butterfly's own terms, the camera following the butterfly throughout.

Insert:

movie03a.jpg

movie03b.jpg

\section{Movie 04: Graphix}

Graphix is an abstract multi-textured visualisation of style, colour, and contemporary ideas. Images fade in and out, passing forwards and backwards, left and right, up and down creating abstract themes and fast motion.
Insert:
movie04a.jpg
movie04b.jpg
movie04c.jpg 


\section{Movie 05: Motion Static}

Motion Static is a fast paced collection of images based around the theme of electricity and power. Motion plays a large part in the movie and the sharp camera work gives a large industrial feel to the movie.

Insert:

movie05a.jpg

movie05b.jpg

\section{Movie 06: Futurecity (computer animation)}

Futurecity is a 'city' (buildings, vehicles, populations) set in the 'future' (the world of science fiction and fantasy). The movie shows structure and moving shapes all displayed in a computer animated, 3D wire frame. This isn't a realistic movie, more of an abstract view of a futuristic metropolis that one travels through on a flying camera.

Insert:

movie06a.jpg

movie06b.jpg

movie06c.jpg

movie06d.jpg 